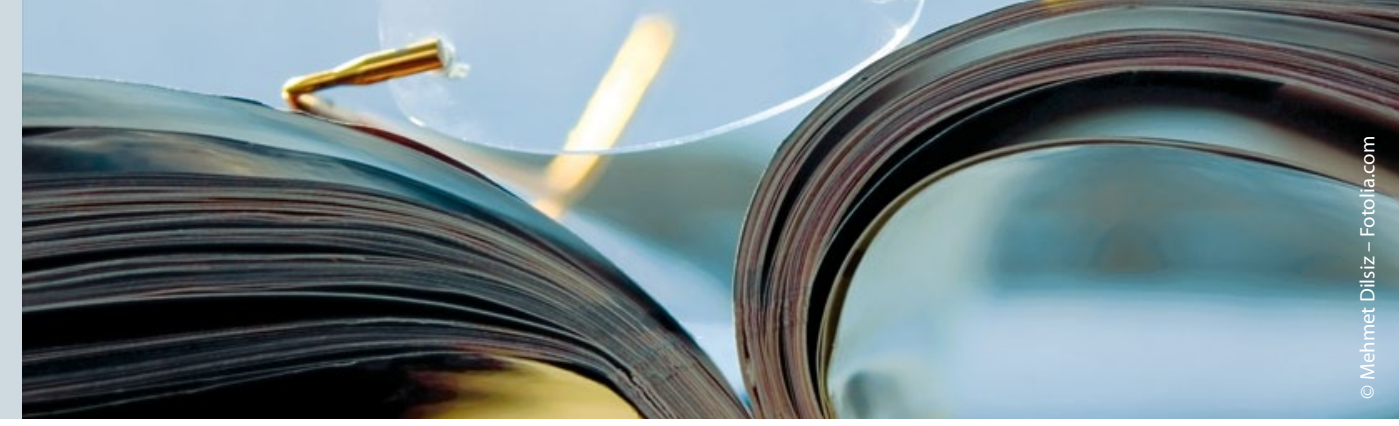

derung der Lautstärke festgestellt. Signifikante Ergebnisse zeigten sich auch in objektivierenden Parametern wie auditorische kortikale evozierte Potentiale in der Magnet-Enzephalografie. Die Autoren schließen daraus, dass die Lautstärke eines Tinnitus durch eine einfache, preiswerte und zudem noch angenehme Art beeinflusst werden kann.

Kommentar: Diese Studie präsentiert ein sensationelles Ergebnis, denn erstmals ist es in einem randomisierten Verfahren gelun- gen, chronischen Tinnitus signifikant zu beeinflussen. Der Aufwand für die Patienten ist dabei minimal. Allenfalls die Herstellung der maßgeschneiderten Musik erfordert technischen Aufwand. Es muss hierbei berücksichtigt werden, dass dieses Verfahren nur bei Patienten angewendet worden ist, die einen chronischen Tinnitus mit vermuteter kortikaler Überaktivität aufwiesen. Andere Tinnitusformen wie zum Beispiel periphere Formen oder pulssynchrone Tinnitusformen sind hierdurch wahrscheinlich nicht zu beeinflussen. Die Studie muss nun an einem größeren Kollektiv überprüft werden. Die Arbeitsgruppe hat bereits die Ergebnisse in weiteren Untersuchungen bestätigen können.

Prof. Dr. Stefan Evers
Okamoto $\mathrm{H}$ et al. Listening to tailor-made notched music reduces tinnitus loudness and tinnitus-related auditory cortex activity. Proc Natl Acad Sci USA 2010; 107: 1207-10

\title{
Amusie nach Schlaganfall
}

In mehreren Studien ist bislang systematisch untersucht worden, inwieweit es nach einem Schlaganfall zu amusischen Störungen kommt. Hierunter versteht man Störungen der Musikwahrnehmung oder der Musikausübung durch eine umschriebene Hirnläsion. Es ist noch wenig darüber bekannt, wie sich diese amusischen Störungen wieder erholen und welche Auswirkungen die verschiedenen Lokalisationen der Hirnläsion haben können.

n dieser Studie sind Schlaganfallpati-

enten mit einem Infarkt im Gebiet der

A. cerebri media rechts oder links eine Woche, drei Monate und sechs Monate nach dem Schlaganfall mittels eines standardisierten Tests für Amusie untersucht worden. Außerdem wurden zu den Untersuchungszeitpunkten magnetenzephalografische Studien durchgeführt, um die sogenannte Mismatch Negativität (MMN) zu ermitteln.

\section{Störung bei rechtshemisphärischen Infarkten stärker ausgeprägt}

Insgesamt konnten 24 Patienten mit einem linkshemisphärischen und $29 \mathrm{~Pa}$ tienten mit einem rechtshemisphärischen Schlaganfall eingeschlossen werden. Amusische Störungen waren bei rechts- hemisphärischen Infarkten stärker ausgeprägt als bei linkshemisphärischen. Das Ausmaß der Amusie korrelierte dabei mit der Stärke der MMN. Im Verlauf über sechs Monate waren die amusischen Störungen insgesamt rückläufig. Interessanterweise zeigten die amusischen Patienten, die auch eine Läsion im auditorischen Kortex hatten, eine deutlich schlechtere Erholung ihrer Amusie. Weiterhin wurden andere neuropsychologische Testverfahren durchgeführt. Hier zeigte sich, dass die amusischen Patienten auch im Arbeitsgedächtnis, in der Aufmerksamkeit und in der kognitiven Flexibilität schlechter abschnitten als Patienten ohne Amusie. Die Autoren schlossen daraus, dass einer Amusie nach einem Schlaganfall im Allgemeinen primäre Mechanismen der kognitiven Beeinträchtigung zugrunde liegen und dass auditorische Probleme nur dann dazu beitragen, wenn auch der auditorische Kortex mitbetroffen ist.

Kommentar: Diese höchst aufwendige Studie zeigt einmal mehr, dass sich amusische Störungen nach einem Schlaganfall einstellen und auch wieder zurückentwickeln können. Die Korrelation mit Aufmerksamkeitsfunktionen ist hier überzeugend mittels Magnetenzephalografie gezeigt worden. Nicht evaluiert werden konnte jedoch in dieser Studie, welche Faktoren auf die Rückbildung der amusischen Störungen Einfluss nehmen. Dies ist von besonderem Interesse in der Rehabilitation von Schlaganfallpatienten, die unter ihren amusischen Störungen leiden oder bei denen der Einsatz von Musik zum Beispiel auch in der Logopädie sinnvoll ist. Hier müssten dringend prospektive Therapiestudien durchgeführt werden.

Prof. Dr. Stefan Evers

Särkämö T et al. Auditory and cognitive deficits associated with acquired amusia after stroke: a magnetoencephalography and neuropsychological follow-up study. PLoS One 2010; 5: e15157 